\title{
Genetic variations and drug repurposing provides key insights into the disruption of the SARS COV2
}

\author{
Gayatri Panda ${ }^{1 \#}$, Neha Mishra ${ }^{1 \#}$ and Arjun Ray ${ }^{1 *}$ \\ ${ }^{1}$ Department of Computational Biology, Indraprastha Institute of Informa- \\ tion Technology, Okhla, India \\ * E-mail: Correspondence to Arjun Ray (arjun@iiitd.ac.in) \\ \#Authors contributed equally
}

\begin{abstract}
The severe acute respiratory syndrome coronavirus 2 (SARS-CoV2 ) is mediated via the initial interaction of the virus' spike (S-) protein with a human receptor protein called angiotensin-converting enzyme 2 (hACE2). Interference in this association can have immense therapeutic importance. We used an in - silico combinatorial approach involving homology-based protein modeling, protein-protein docking, binding energy estimation and virtual screening techniques to probe for genetic variations and drug molecules for disruption of the hACE2-CoV2 interaction. Our findings identified Ser19Pro variation on hACE2 showing similar structural stability to the native protein while having a destabilizing effect in the hACE2 $2_{\text {Ser } 19 P r o}$-Sprotein complex. We also found several FDA-approved drug molecules that can potentially induce competition-mediated destabilization of the hACE2-CoV2 complex. In conclusion, these findings provide critical insights for intervention strategies targeting the pathogenicity of SARS-CoV-2.
\end{abstract}

\section{Introduction}

The global pandemic of severe acute respiratory syndrome coronavirus 2 (SARS-CoV-2) has spread to more than 200 countries and infected more than 13 million people worldwide and killed more than 70,000 (W.H.O.). Human-to-human transmission of this disease primarily occurs through close contact and through respiratory droplets. Coronaviruses (CoVs) have a single-stranded positive-sense RNA genome encapsulated within a glycoprotein studded membrane envelope, which gives it a crown-like appearance (Goldsmith et al., 2004). Due to absence of any clinically approved vaccines 
or drugs that specifically target SARS-CoV2 proliferation mechanism, this infection is spreading at an exponential rate.

The betacoronavirus genome encodes several structural (surface-located spike (S) glycoprotein) and non-structural proteins (RNA-dependent RNA polymerase ( $\mathrm{RdRp})$, coronavirus main protease (3CLpro), and papain-like protease (PLpro) (Wu et al. (2020)). The S protein present in both SARS$\mathrm{CoV}$ and SARS-CoV-2 mediates in host cell invasion via binding to the human receptor Angiotensin Converting Enzyme - 2 (hACE2)(Du et al. (2009); Hamming et al. (2004); Harmer et al. (2002)). hACE2 is present on the outer surface of cells of lungs, kidneys, arteries, etc., and is responsible for raising the blood pressure by binding to the Angiotensin receptors (Warner et al. (2005); Chappell (2010)). The binding affinity of SARS-CoV2 with hACE2 is 10-20 times higher than the S-protein of SARS-Cov, causing it to be more contagious and transmissible(Wan et al. (2020)). In-vitro studies have also shown a strong correlation between the expression of hACE2 and the infection of the SARS-CoV (Cao et al. (2020); Hofmann et al. (2004); Li et al. (2007)).

In order to understand the structural basis of recognition by SARVCoV2 and hACE2, a study was performed by Shang et., al. (Shang et al. (2020)), in which a chimeric receptor-binding domain (RBD) was designed, that uses the core from SARS-CoV RBD as the crystallization scaffold and the receptor-binding motif (RBM) from SARSCoV-2 as the functionally relevant unit. They reported that SARS-CoV2 has a larger binding interface and thus forms more contact with hACE2 as compared to SARS-Cov. The conformation of loop of SARS-CoV2 RBM in hACE2 binding ridge is different from SARS-Cov. It has a four residue motif glycine valine/glutamineglutamate/threonine-glycine; two relatively bulky residues and two flexible glycines allow the loop to take a different conformation, which is not there in SARS-Cov. Due to these structural differences, a new hydrogen bond between Asn487 and Ala475 in SARS-CoV-2 RBM is formed moving the loop (containing Ala-475) closer to hACE2 and thus forming more contacts with the N-terminal helix of hACE2. Also, Phe486 (SARS-CoV2) forms a hydrogen bond with hACE2 and inserts into a hydrophobic pocket in hACE2 formed by residues Met82, Leu79, and Tyr83, found to be favourable for hACE2 binding. At the interface between SARS-CoV2 and hACE2, there are two virus binding hotspots, hotspot 31(hACE2 -Lys31) and hotspot 353(hACE2-Lys353). These two hotspots undergo structural change in their conformation due to reduced support from RBD residues (Leu455 and Asn501), leading to more contact with main chain SARS-CoV2 and increase in stability of binding interface.

In a paper published by Cao et. al., functional coding variants of hACE2 
and their Allele Frequency (AF) in different populations was investigated (Cao et al. (2020)). In their study, they had taken ChinaMAP (China Metabolic Analytics Project, under review) and 1000 genome databases and found 62 coding variants out of which 32 variants were found to affect aminoacid sequence. They had reported seven hotspot missense mutations, in different populations: Lys26Arg, Ile468Val, Ala627Val, Asn638Ser, Ser692Pro, Asn720Asp, and Leu731Ile/Leu731Phe, of hACE2 . Six low-frequency loci (rs200180615, rs140473595, rs199951323, rs147311723, rs. 149039346, and rs73635825) were found to be specific in the 1000 Genomes Project database (Consortium et al. (2015)) but none of the variations resided at the binding site hACE2 and SARS-CoV2.

The previous studies clarified that the structural features of SARS-CoV$2 \mathrm{RBM}$, including the hACE2-binding ridge and the hotspots-stabilizing residues, all contribute critically to the high hACE2-binding affinity of SARS$\mathrm{CoV}-2$. Hence, any genetic variation in these interacting residues of hACE2 and SARS-CoV2 may possibly affect its binding. In order to understand how variations in these interacting residues of hACE2 can affect its binding with SARS-CoV2, only the missense variations in residues involved in binding with SARS-CoV2 were taken into consideration. This paper deciphers the interaction between hACE2 -SARS CoV2 in its native and variant structures, using molecular docking technique. It also aims to identify FDAapproved drugs that can be repurposed with the potential to affect binding of hACE2-CoV2.

\section{Materials and Methods}

\section{Modeling the population variants}

For modeling of the hACE2 genetic variants, residues forming the interface surfaces of the hACE2 and RBM of SARS-CoV-2 were firstly identified from the recently published crystal structure (PDBID: 6vw1)(Shang et al. (2020)) using PIC web server (Tina et al. (2007)). Also, the complete list of the global population variations for the hACE2 gene was retrieved from the gnomAD(Karczewski et al. (2020)) and dbSNP (Sherry et al. (1999)) databases. These were further filtered, based upon the residues lying at the interface of hACE2-CoV2 complex. The variant models were generated using Modeller v9.10(Šali and Blundell (1993)) using the native hACE2 from the crystal structure as template and were finally refined using the stand-alone version of ModRefine(Xu and Zhang (2011)). The quality of the models were assessed using ProQ2 (Ray et al. (2012)). 


\section{Docking for studying the effect of genetic variations on hACE2-CoV2 interaction}

The CoV2 S-protein structure from the crystal structure (PDBID: 6vw1), along with the native (as a control experiment) hACE2 and its variants were submitted for protein-protein docking, using HADDOCK web server(Dominguez et al. (2003)). The identified interface residues of the proteins were provided as active residues while the surrounding were defined as passive. The docked structures were clustered and ranked according to the HADDOCK score, where the score is defined as: 1.0 Evdw +0.2 Eelec +1.0 Edesol +0.1 EAIR . The HADDOCK score is an arbitrary unit consisting of a combination of energy terms (VDW, electrostatics), along with empirical terms (desolvation, buried surface area) and restrained violation energies.

\section{High-throughput virtual screening of FDA approved drugs}

Virtual screening was performed against the native hACE2 structure of 6vw1 using AutoDock (Morris et al. (2009)). 358 human FDA-approved drug molecules were downloaded from the ZINC database (Irwin and Shoichet (2005)). The sampling region was defined around the interface residues previously identified. We selected the enhanced-performancing Lamarckian Genetic Algorithm (LGA) for ligand conformational searching. For each drug molecule, the docking parameters were as follows: trials of 20 hybrid GA-LS runs, population size of 150, random starting position, orientation and conformation, translation step ranges of 1.5 , mutation rate of 0.02 , crossover rate of 0.8 , local search rate of 0.06 , and 1750000 energy evaluations. A ranked cluster analysis was performed at the end of the run and the cluster with the highest number of members was chosen for identifying the best docked conformation.

\section{Analysis}

Structural stability of the variant as well as native structures was calculated using FoldX where the Gibbs free energy of folding is in $\mathrm{kcal} / \mathrm{mol}$ (Schymkowitz et al. (2005)).

The fraction of contacts between the hACE2 native and variants with Cov2 at ther binding interface was calculated and compared using PIC webserver (Tina et al. (2007)) and further processed using in-house scripts.

The structural figures were created using UCSF Chimera (Pettersen et al. (2004)) and Pymol (DeLano (2002)). 


\section{Results and Discussion}

\section{Deciphering the effect of population variations on the hACE2-CoV-2 interaction}

Host invasion of the SARS-CoV-2 is mediated via the initial recruitment of the virus' spike (S) protein by the receptor protein hACE2, followed by the transmembrane protease, serine 2 (TMPRSS2) cleaving the protein $\mathrm{S}$ at the S1/S2 and the $\mathrm{S} 2$ sites, resulting in the fusion of the viral into the cellular membrane (Asselta et al. (2020); Hoffmann et al. (2020)). To decipher the molecular insights of the pathogenicity of the SARS-CoV2, the key residues stabilizing the interaction of the complex were identified using Protein Interaction Calculator (PIC) (Tina et al. (2007)) from the crystal structure (PDBID: 6vw1) (Shang et al. (2020)). The structures revealed residues Ser19, Gln24, Phe28, Lys31, Glu35, Glu37, Asp38, Tyr41,Gln42, Leu79, Met82,Tyr 83, Glu329 and Lys353 of the hACE2 interacting noncovalently with residues Arg439, Tyr449, Ala475, Glu484, Phe486, Asn487, Tyr489, Gln493, Lys496, Gln498, Thr500, Asn501, Gly502 and Tyr505 of the RBM domain of the CoV2 S-protein. The residues resulted in a highly intricate network of 41 pairs of interactions where there were 5 pairs of hydrophobic interactions, 30 pairs of hydrogen bonds, 3 ionic interacting pairs, 2 aromatic-aromatic residue interactions and 1 aromatic-sulphur interaction (Figure 1A)(Supplementary T1).

With this information of the key residues, we investigated the role of global genetic variations at the interaction site of the hACE2-CoV2. While several studies have been performed to find the genetic diversity of the SARSCoV2 (Phan (2020); Wang et al. (2020a); Tang et al. (2020); Wertheim (2020); Sardar et al. (2020)), very few studies have studied the human genetic population variations for the key interacting proteins for the proliferation of the disease (Asselta et al. (2020); Cao et al. (2020); Li et al. (2005)) and none have reported on the interface site of the virus-human interaction. Hence, to investigate the role of population variations on the hACE2-S-protein interacting site, we retrieved all the variations from the gnomAD(Karczewski et al. (2020)) and dbSNP (Sherry et al. (1999)) database for the European, African, African American, Asian, East Asian, Other Asian, Latin American and South Asian populations. Mapping these variations with our identified residues, we found five low-frequency loci - rs73635825 (Ser19Pro), rs778500138 (Glu35Asp), rs146676783 (Glu37Lys), rs766996587 (Met82Ile) and rs143936283 (Glu329Gly) (Supplementary T2). All of these variations had an AF of less than 0.001. Interestingly, variations rs73635825 (Ser19Pro) and rs143936283 (Glu329Gly) were present only in the European population, 
an epicenter of the current pandemic. Curiously, 4 out of the 5 residues (F28, L79, M82 and Y83) from a recent publication identifying key residues in hACE2 for interaction mechanism of SARS-CoV-2, had population variations, as seen in our analysis(Wang et al. (2020b)).

These variations were then modeled for studying the implications to its binding to CoV2 protein using homology modeling techniques. The structure of hACE2 from the crystal structure was used for template and using Modeller (Šali and Blundell (1993)), the variant models were built. Using ModRefiner (Xu and Zhang (2011)), the models were further refined and ProQ2 was used for the quality assessment of the identification of the final structures (Ray et al. (2012)). Analysis of the Gibbs free energy of folding for the structures revealed that apart from Ser19Pro (Gibbs free energy of folding $=-91.46 \mathrm{kcal} / \mathrm{mol}$ ), all the final refined models of the variants showed a higher structural stability, in comparison to the native hACE2 structure (Gibbs free energy of folding $=-91.31 \mathrm{kcal} / \mathrm{mol})($ Figure 1B).

Next, to study the molecular interaction of these variants, we employed state-of-the-art protein-protein docking method, HADDOCK, where these final refined variant structures were computationally sampled for their interaction with the CoV2 S-protein. Performing a control experiment by docking the native hACE2 structure with the S-protein, we found a gradient of stabilities for the bound variant complexes (Figure 1C). While the control experiment revealed the binding score of native hACE2 with nCOV2 being around -91, Glu35Asp and Met82Ile variant models had a much higher binding score (See Materials and Methods for the details on the scoring scheme) with nCOv2 ( -110 and -101, respectively). Ser19Pro variation resulted in destabilizing the complex while variants Glu329 and Glu37Lys had similar binding strength, compared to the native hACE2 interaction with CoV2. To understand the variant complex's interaction in-depth, we further analyzed the fraction of native contact pairs present in the complexes (Figure 1D). Interestingly, the analysis revealed that, apart from the Ser19Pro variation, all the others displayed very low conservation of the native interacting pairs. Also, the relative orientation of all the variations (except in Ser19Pro) indicated a complete rewiring of the protein-protein interaction network (Supplementary Figure S1). 

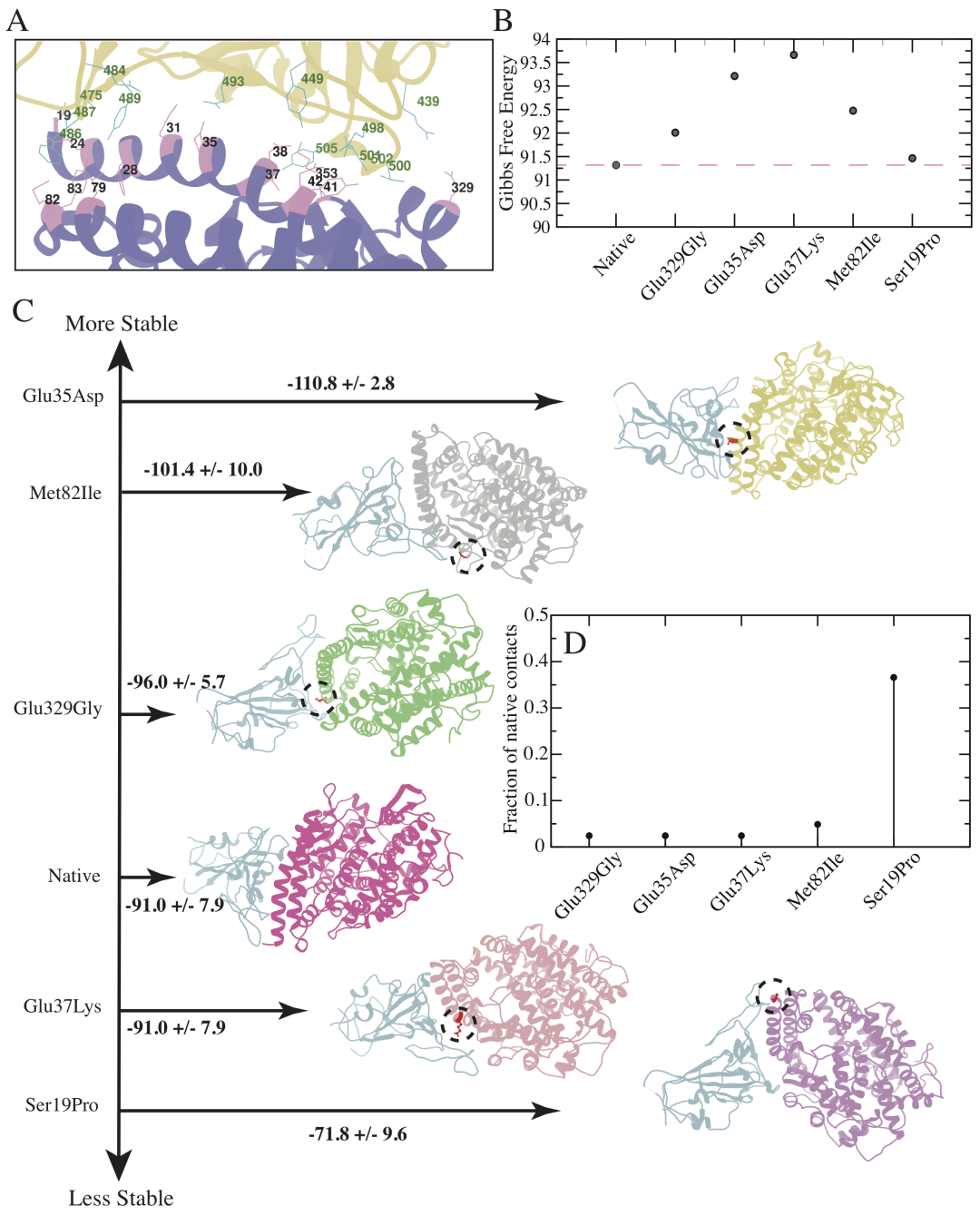

Figure 1: Deciphering the role of genetic variations for the hACE2CoV2 complex: A) Highlighting the interacting residues involved in the native complex of hACE2 (coloured in pink and labelled in black) with RBD of CoV2 (coloured in cyan and labelled in green) in the crystal structure $6 \mathrm{vw} 1$. B) Structural stability of the native hACE2 and its variant models were calculated using FoldX to estimate the Gibbs free energy of folding. C) The interaction of the native and variant hACE2 structures with CoV2 was studied using HADDOCK webserver. RBD of the CoV2 is coloured in light blue while the various hACE2 structures are represented in different colours. The scores (marked on the arrows) are in HADDOCK scoring scale (See Materials and Methods). D. Fraction of native contacts were calculated by analyzing the presence of interacting residue pairs between hACE2 and CoV2 crystal structure, in the variantycomplexes. 


\section{Virtual screening of FDA-approved drugs at the hACE2 interacting site}

Drug repurposing is emerging as an alternative, albeit effective approach in combating various diseases. The immense importance of this approach can be highlighted, even in the present pandemic of SARS-CoV-2, as inferred from the sudden augmentation in the demand and consumption of hydroxychloroquine, an antimalarial drug in COVID-19 infected patients. We next asked, if any of the FDA-approved drug could potentially and effectively interact with hACE2 at the same site as that of SARS-CoV-2 spike protein, and therefore, could potentially induce competition-mediated destabilization of the hACE2-CoV2 complex. To validate this hypothesis, we employed virtual screening techniques using AutoDock software (Morris et al. (2009)). 358 molecules from ZINC database (Irwin and Shoichet (2005)), mapping onto 328 DrugIDs were screened against the native hACE2 (Supplementary Table 3). Notably, the docking space was restricted to the interacting region of the protein. For each testing molecule, a random starting position, orientation and conformation was taken and with exhaustive energy evaluations, 20 hybrid GA-LS runs were performed. Analysis of the best binding conformation from the largest cluster for each drug molecule revealed larger variation in the binding energy, varying from -9 to $-4.5 \mathrm{kcal} / \mathrm{mol}$, with maximum reaching to -19 kcal/mol (Figure 2A). In-depth analysis and manual inspection further revealed that the majority of the top ranked docked poses were buried in the core of the protein with strong binding energy (Supplementary figure S2), indicating potential regulators for the protein.

We further screened for conformation of the molecules for those encompassing the interface of hACE2-CoV2 (Figure 2B). Collectively, our results suggest that a handful of FDA approved drugs, namely Gentamicin, Natamycin, Nisoldipine, Saquinavir and Linagliptin lay at the interface of the hACE2 and could potentially disrupt the viral-host protein interaction by potentially serving as competitive inhibitor (Supplementary Table 3). Gentamicin is part of broad-spectrum antibiotics, Natamycin is an antifungal antibiotic, Nisoldipine is a 1,4-dihydropyridine calcium channel blocker and used for the treatment of hypertension, Saquinavir is HIV protease inhibitor while Linagliptin is a DPP-4 inhibitor and used in for the treatment of type II diabetes. 
A

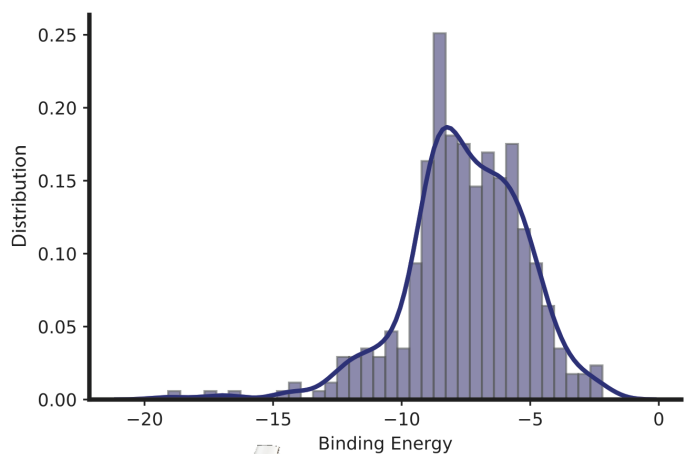

B

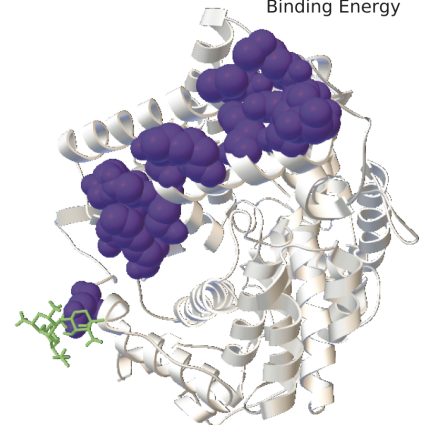

$\mathrm{C}$

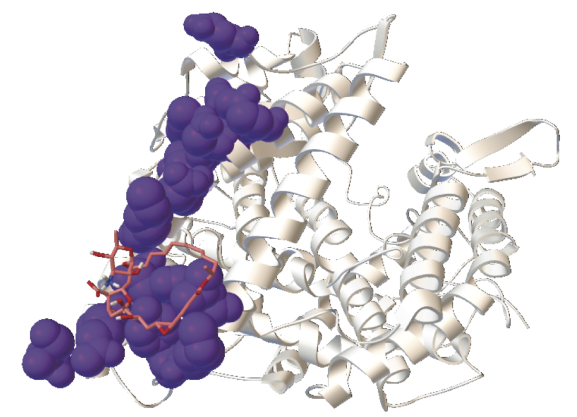

D
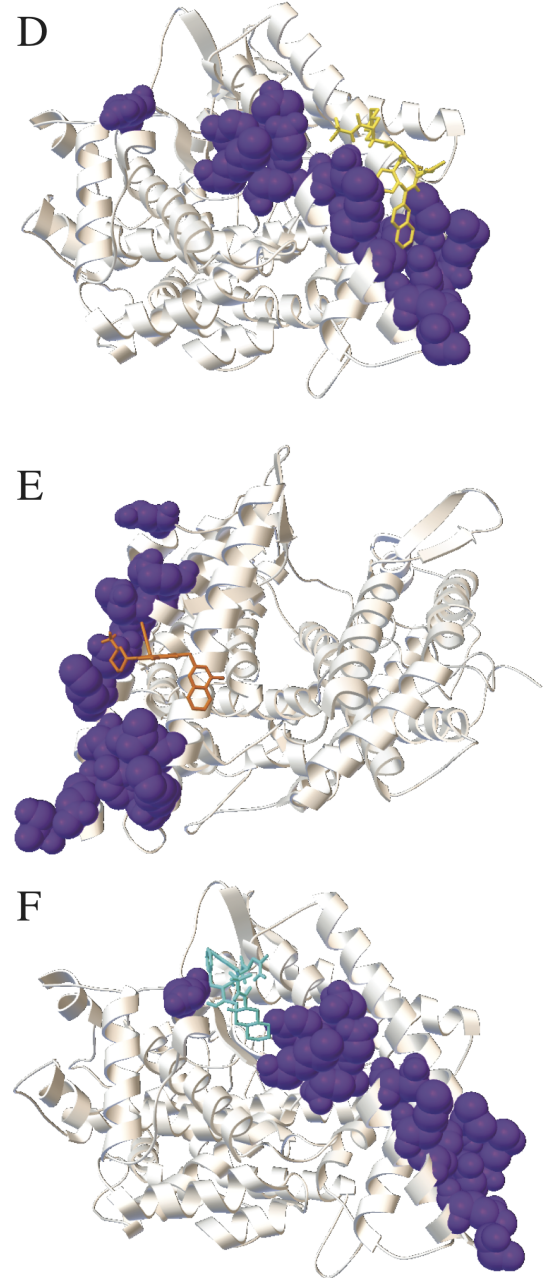

Figure 2: Probing the protein-protein binding interface of hACE2 with FDA-approved drug molecules: Probing the protein-protein binding interface of hACE2 with FDA-approved drug molecules: (A) Distribution of the binding en- ergies of 358 drug molecules that were virtually screened using AutoDock. (B-F) Snapshot of the drug-protein interaction on the interface residues (rep- resented in purple spheres) while the drug molecule is represented in wire frame. The best binding molecules represented here are: B) Gentamicin $(-15.18 \mathrm{kcal} / \mathrm{mol})$; green, C) Natamycin $(-11.04 \mathrm{kcal} / \mathrm{mol})$; red D) Nisoldipine $(-9.07 \mathrm{kcal} / \mathrm{mol})$; yellow E) Saquinavir $(-8.34 \mathrm{kcal} / \mathrm{mol})$; pink and F) Linagliptin $(-7.72 \mathrm{kcal} / \mathrm{mol})$; cyan. 


\section{Conclusion}

In this study, we have investigated the role of genetic variations and screened for FDA-approved drugs for disrupting the binding of hACE2-CoV2. To the best of our knowledge, this is the first report which highlights how population variations can lead to complete rewiring of the virus' protein-protein interaction network and identify novel therapeutically important insights. Study of the genetic variations of the hACE2 at the interacting site of S-protein revealed that while Ser19Pro variation in hACE2, which had a very similar structural stability as to the native hACE2 and showed a similar orientation and interacting network as compared to the native, showed a destabilized complex with the virus's protein. This makes the variant a promising candidate for future gene-editing based therapeutics. Also, with the hypothesis of induced competition-mediated destabilization of the hACE2-CoV2 complex, we explored the possibility of repurposing of FDA-approved drug molecules. We found five drugs, namely, Gentamicin, Natamycin, Nisoldipine, Saquinavir and Linagliptin strongly binding at the virus interaction interface of hACE2. However, these results merit a competitive-binding assay experiment for validating these findings. This study highlights novel genetic and pharmaceutical insights into the SARS-CoV2's mitigation and shall provide key insights for the future experimental validation for administering drugs against the virus.

\section{Acknowledgements}

The authors would like to thank Dr. Gaurav Ahuja and Dr. Debarka Sengupta at IIIT Delhi as well as Dr. Shantanu Sengupta, Dr. Deepanjan Paul and Dr. Vinod Scaria from CSIR-IGIB for their critical insights into shaping the manuscript. We would also like to thank the HPC facility at IIIT Delhi for facilitating the necessary computing resources.

\section{References}

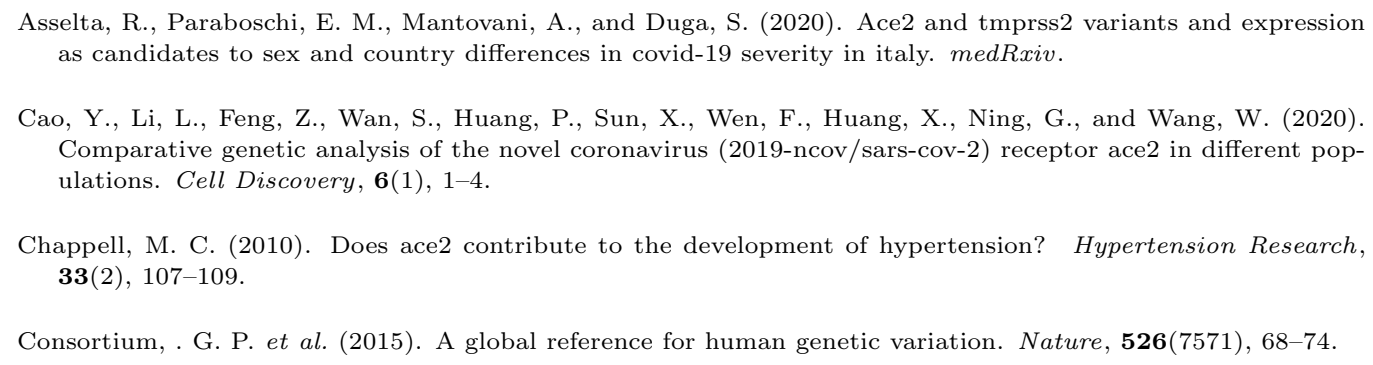


DeLano, W. L. (2002). The pymol molecular graphics system. http://www. pymol. org.

Dominguez, C., Boelens, R., and Bonvin, A. M. (2003). Haddock: a protein- protein docking approach based on biochemical or biophysical information. Journal of the American Chemical Society, 125(7), 1731-1737.

Du, L., He, Y., Zhou, Y., Liu, S., Zheng, B.-J., and Jiang, S. (2009). The spike protein of sars-cova target for vaccine and therapeutic development. Nature Reviews Microbiology, 7(3), 226-236.

Goldsmith, C. S., Tatti, K. M., Ksiazek, T. G., Rollin, P. E., Comer, J. A., Lee, W. W., Rota, P. A., Bankamp, B., Bellini, W. J., and Zaki, S. R. (2004). Ultrastructural characterization of sars coronavirus. Emerging infectious diseases, $\mathbf{1 0}(2), 320$.

Hamming, I., Timens, W., Bulthuis, M., Lely, A., Navis, G., and van Goor, H. (2004). Tissue distribution of ace2 protein, the functional receptor for sars coronavirus. a first step in understanding sars pathogenesis. The Journal of Pathology: A Journal of the Pathological Society of Great Britain and Ireland, 203(2), $631-637$.

Harmer, D., Gilbert, M., Borman, R., and Clark, K. L. (2002). Quantitative mrna expression profiling of ace 2, a novel homologue of angiotensin converting enzyme. FEBS letters, 532(1-2), 107-110.

Hoffmann, M., Kleine-Weber, H., Schroeder, S., Krüger, N., Herrler, T., Erichsen, S., Schiergens, T. S., Herrler, G., Wu, N.-H., Nitsche, A., et al. (2020). Sars-cov-2 cell entry depends on ace 2 and tmprss 2 and is blocked by a clinically proven protease inhibitor. Cell.

Hofmann, H., Geier, M., Marzi, A., Krumbiegel, M., Peipp, M., Fey, G. H., Gramberg, T., and Pöhlmann, S. (2004). Susceptibility to sars coronavirus s protein-driven infection correlates with expression of angiotensin converting enzyme 2 and infection can be blocked by soluble receptor. Biochemical and biophysical research communications, $\mathbf{3 1 9}(4)$, 1216-1221.

Irwin, J. J. and Shoichet, B. K. (2005). Zinc- a free database of commercially available compounds for virtual screening. Journal of chemical information and modeling, 45(1), 177-182.

Karczewski, K. J., Francioli, L. C., Tiao, G., Cummings, B. B., Alföldi, J., Wang, Q., Collins, R. L., Laricchia, K. M., Ganna, A., Birnbaum, D. P., Gauthier, L. D., Brand, H., Solomonson, M., Watts, N. A., Rhodes, D., Singer-Berk, M., England, E. M., Seaby, E. G., Kosmicki, J. A., Walters, R. K., Tashman, K., Farjoun, Y., Banks, E., Poterba, T., Wang, A., Seed, C., Whiffin, N., Chong, J. X., Samocha, K. E., Pierce-Hoffman, E., Zappala, Z., O'Donnell-Luria, A. H., Vallabh Minikel, E., Weisburd, B., Lek, M., Ware, J. S., Vittal, C., Armean, I. M., Bergelson, L., Cibulskis, K., Connolly, K. M., Covarrubias, M., Donnelly, S., Ferriera, S., Gabriel, S., Gentry, J., Gupta, N., Jeandet, T., Kaplan, D., Llanwarne, C., Munshi, R., Novod, S., Petrillo, N., Roazen, D., Ruano-Rubio, V., Saltzman, A., Schleicher, M., Soto, J., Tibbetts, K., Tolonen, C., Wade, G., Talkowski, M. E., , Neale, B. M., Daly, M. J., and MacArthur, D. G. (2020). The mutational constraint spectrum quantified from variation in 141,456 humans. bioRxiv.

Li, W., Zhang, C., Sui, J., Kuhn, J. H., Moore, M. J., Luo, S., Wong, S.-K., Huang, I.-C., Xu, K., Vasilieva, N., et al. (2005). Receptor and viral determinants of sars-coronavirus adaptation to human ace2. The EMBO journal, 24(8), 1634-1643.

Li, W., Sui, J., Huang, I.-C., Kuhn, J. H., Radoshitzky, S. R., Marasco, W. A., Choe, H., and Farzan, M. (2007). The s proteins of human coronavirus nl63 and severe acute respiratory syndrome coronavirus bind overlapping regions of ace 2 . Virology, $\mathbf{3 6 7}(2), 367-374$.

Morris, G. M., Huey, R., Lindstrom, W., Sanner, M. F., Belew, R. K., Goodsell, D. S., and Olson, A. J. (2009). Autodock4 and autodocktools4: Automated docking with selective receptor flexibility. Journal of computational chemistry, 30(16), 2785-2791.

Pettersen, E. F., Goddard, T. D., Huang, C. C., Couch, G. S., Greenblatt, D. M., Meng, E. C., and Ferrin, T. E. (2004). Ucsf chimeraa visualization system for exploratory research and analysis. Journal of computational chemistry, 25(13), 1605-1612.

Phan, T. (2020). Genetic diversity and evolution of sars-cov-2. Infection, Genetics and Evolution, 81, 104260.

Ray, A., Lindahl, E., and Wallner, B. (2012). Improved model quality assessment using proq2. BMC bioinformatics, 13(1), 224. 
Šali, A. and Blundell, T. L. (1993). Comparative protein modelling by satisfaction of spatial restraints. Journal of molecular biology, 234(3), 779-815.

Sardar, R., Satish, D., Birla, S., and Gupta, D. (2020). Comparative analyses of sar-cov2 genomes from different geographical locations and other coronavirus family genomes reveals unique features potentially consequential to host-virus interaction and pathogenesis. bioRxiv.

Schymkowitz, J., Borg, J., Stricher, F., Nys, R., Rousseau, F., and Serrano, L. (2005). The foldx web server: an online force field. Nucleic acids research, 33(suppl_2), W382-W388.

Shang, J., Ye, G., Shi, K., Wan, Y., Luo, C., Aihara, H., Geng, Q., Auerbach, A., and Li, F. (2020). Structural basis of receptor recognition by sars-cov-2. Nature, pages 1-8.

Sherry, S. T., Ward, M., and Sirotkin, K. (1999). dbsnpdatabase for single nucleotide polymorphisms and other classes of minor genetic variation. Genome research, 9(8), 677-679.

Tang, X., Wu, C., Li, X., Song, Y., Yao, X., Wu, X., Duan, Y., Zhang, H., Wang, Y., Qian, Z., et al. (2020). On the origin and continuing evolution of sars-cov-2. National Science Review.

Tina, K., Bhadra, R., and Srinivasan, N. (2007). Pic: protein interactions calculator. Nucleic acids research, 35(suppl 2), W473-W476.

Wan, Y., Shang, J., Graham, R., Baric, R. S., and Li, F. (2020). Receptor recognition by the novel coronavirus from wuhan: an analysis based on decade-long structural studies of sars coronavirus. Journal of virology, 94(7).

Wang, C., Liu, Z., Chen, Z., Huang, X., Xu, M., He, T., and Zhang, Z. (2020a). The establishment of reference sequence for sars-cov-2 and variation analysis. Journal of Medical Virology.

Wang, Q., Zhang, Y., Wu, L., Niu, S., Song, C., Zhang, Z., Lu, G., Qiao, C., Hu, Y., Yuen, K.-Y., Wang, Q., Zhou, H., Yan, J., and Qi, J. (2020b). Structural and functional basis of sars-cov-2 entry by using human ace2. Cell.

Warner, F. J., Lew, R. A., Smith, A. I., Lambert, D. W., Hooper, N. M., and Turner, A. J. (2005). Angiotensinconverting enzyme 2 (ace2), but not ace, is preferentially localized to the apical surface of polarized kidney cells. Journal of Biological Chemistry, 280(47), 39353-39362.

Wertheim, J. O. (2020). A glimpse into the origins of genetic diversity in sars-cov-2. Clinical Infectious Diseases: An Official Publication of the Infectious Diseases Society of America.

(W.H.O.), W. H. O. (2000). Coronavirus disease 2019 (covid-19) situation report 79.

Wu, A., Peng, Y., Huang, B., Ding, X., Wang, X., Niu, P., Meng, J., Zhu, Z., Zhang, Z., Wang, J., et al. (2020). Genome composition and divergence of the novel coronavirus (2019-ncov) originating in china. Cell host $\mathcal{E}$ microbe.

$\mathrm{Xu}$, D. and Zhang, Y. (2011). Improving the physical realism and structural accuracy of protein models by a two-step atomic-level energy minimization. Biophysical journal, 101(10), 2525-2534. 